\title{
Bioinformatics screening of biomarkers related to liver cancer
}

\author{
Ye-Cheng Wang ${ }^{1}$, Zhen-Bo Tian ${ }^{1}$ and Xu-Qing Tang ${ }^{1,2^{*}}$ (1)
}

From Fifteenth International Conference on Intelligent Computing (ICIC 2019)

Nanchang, China. 3-6 August 2019

\author{
*Correspondence: \\ txq5139@jiangnan.edu.cn \\ ${ }^{1}$ School of Science, Jiangnan \\ University, Wuxi 214122, \\ China \\ Full list of author information \\ is available at the end of the \\ article
}

\begin{abstract}
Background: Liver cancer is a common malignant tumor in China, with high mortality. Its occurrence and development were thoroughly studied by high-throughput expression microarray, which produced abundant data on gene expression, mRNA quantification and the clinical data of liver cancer. However, the hub genes, which can be served as biomarkers for diagnosis and treatment of early liver cancer, are not well screened.

Results: Here we present a new method for getting 6 key genes, aiming to diagnose and treat the early liver cancer. We firstly analyzed the different expression microarrays based on TCGA database, and a total of 1564 differentially expressed genes were obtained, of which 1400 were up-regulated and 164 were down-regulated. Furthermore, these differentially expressed genes were studied by using GO and KEGG enrichment analysis, a PPI network was constructed based on the STRING database, and 15 hub genes were obtained. Finally, 15 hub genes were verified by applying the survival analysis method on Oncomine database, and 6 key genes were ultimately identified, including PLK1, CDC20, CCNB2, BUB1, MAD2L1 and CCNA2. The robustness analysis of four independent data sets verifies the accuracy of the key gene's classification of the data set.
\end{abstract}

Conclusions: Although there are complicated differences between cancer and normal cells in gene functions, cancer cells could be differentiated in case that a group of special genes expresses abnormally. Here we presented a new method to identify the 6 key genes for diagnosis and treatment of early liver cancer, and these key genes can help us understand the pathogenesis of liver cancer more deeply.

Keywords: Bioinformatics analysis, Differential expressed genes, PPI, Oncomine, Survival curve analysis

\section{Background}

As one of the common malignant tumors in China, liver cancer accounts for $70-80 \%$ of primary liver malignancies, and are the second leading cause of cancer-related mortality [1-3]. Current treatments for liver cancer mainly include surgery and intervention, author(s) and the source, provide a link to the Creative Commons licence, and indicate if changes were made. The images or other third party material in this article are included in the article's Creative Commons licence, unless indicated otherwise in a credit line to the material. If material is not included in the article's Creative Commons licence and your intended use is not permitted by statutory regulation or exceeds the permitted use, you will need to obtain permission directly from the copyright holder. To view a copy of this licence, visit http:// creativecommons.org/licenses/by/4.0/. The Creative Commons Public Domain Dedication waiver (http://creativecommons.org/publi cdomain/zero/1.0/) applies to the data made available in this article, unless otherwise stated in a credit line to the data. 
etc. However, as the underlying mechanism of liver cancer is still unclear, liver cancer has a high incidence, strong invasiveness and poor prognosis. Data show that about 70\% of liver cancers relapse within 5 years after resection or ablation [4]. Early detection and treatment can effectively improve the prognosis of tumor patients and reduce the mortality rate, but there are usually no obvious clinical symptoms in the early stage of liver cancer. At present, the commonly used diagnostic methods of liver cancer, such as serological tumor markers and imaging techniques, are not satisfactory in clinical effect. Therefore, screening markers related to early diagnosis, invasion and metastasis of liver cancer is of great significance for improving the therapeutic effect and prognosis of liver cancer [5].

In this study, the original data was downloaded from the TCGA database, and the $\mathrm{R}$ software was used to screen the differentially expressed genes (DEGs) in the gene expression profile of liver cancer tissues and adjacent tissues. Using bioinformatics technology to analyze DEGs, combined with Oncomine database to screen potential molecular targets suitable for early diagnosis and immunotherapy of liver cancer [6]. Survival analysis and literature mining were used to verify our results and provided a theoretical basis for further research. Finally, we constructed a clinical prediction model to get more convincing results.

\section{Results}

According to the mRNAs matrix data of normal tissues and liver cancer tissues in the TCGA database, using the $\mathrm{R}$ toolkit, letting $\alpha=2$ and $\beta=0.05$, a total of 1564 DEGs were obtained, of which 1400 were up-regulated and 164 were down-regulated.

1564 DEGs were enriched by GO and KEGG with DAVID, where $\beta=0.01$ was included as inclusion criteria in $\mathrm{GO}$, and $p$ value $<0.05$ was included as inclusion criteria in KEGG. The results of GO enrichment analysis indicated that the DEGs were mainly enriched in BPs, including sister chromatid cohesion and chemical synaptic transmission. CC showed that the DEGs were significantly enriched in extracellular region, chromosome, extracellular space, plasma membrane and anchored component of membrane, and centromere region. MF showed that the DEGs were significantly enriched in hormone activity, sequence-specific DNA binding, growth factor activity and calcium ion binding. The results of KEGG pathway enrichment analysis showed that the DEGs were mainly enriched in Cell cycle, Neuroactive ligand-receptor interaction, Hypertrophic cardiomyopathy, Mineral absorption, Dilated cardiomyopathy, Adrenergic signaling in cardiomyocytes and Cardiac muscle contraction (Seen in Table 1).

The STRING database was used to construct a protein-regulated interaction network diagram and 1498 protein interaction network nodes were obtained. Analyze these nodes to further understand the regulatory relationship between DEGs and proteins in liver cancer tissues and normal tissues. Import the data downloaded in the STRING database into Cytoscape software for visualization, and get the PPI network diagram of DEGs. The degree of CytoHubba plug-in was used to determine the top 15 hub genes for further analysis. They were GNGT1, GNG4, LPAR3, BDKRB1, PLK1, CDC20, CCNB2, ADCY8, BUB1, MAD2L1, AURKB, AGTR2, RLN3, CCNA2, and CENPE.

The expression levels of these 15 hub genes were analyzed in the Oncomine database, and we obtained 6 key genes, PLK1, CDC20, CCNB2, BUB1, MAD2L1 and 
Table 1 Significantly enriched GO terms and KEGG pathways of DEGs

\begin{tabular}{lllcl}
\hline Category & Term & Description & Count & FDR/p value \\
\hline BP term & GO:0007268 & Chemical synaptic transmission & 44 & $2.26 e-04$ \\
BP term & GO:0007062 & Sister chromatid cohesion & 24 & $4.38 e-03$ \\
CC term & GO:0005576 & Extracellular region & 198 & $2.66 e-08$ \\
CC term & GO:0005615 & Extracellular space & 158 & $1.08 e-04$ \\
CC term & GO:0031225 & Anchored component of membrane & 26 & $1.86 e-03$ \\
CC term & GO:0005886 & Plasma membrane & 386 & $1.16 e-02$ \\
CC term & GO:0000775 & Chromosome, centromeric region & 16 & $2.77 e-02$ \\
MF term & GO:0043565 & Sequence-specific DNA binding & 79 & $2.65 e-06$ \\
MF term & GO:0005179 & Hormone activity & 24 & $4.46 e-04$ \\
MF term & GO:0005509 & Calcium ion binding & 88 & $6.24 e-03$ \\
MF term & GO:0008083 & Growth factor activity & 29 & $3.83 e-02$ \\
KEGG pathway & hsa04080 & Neuroactive ligand-receptor interaction & 46 & $2.33 e-07$ \\
KEGG pathway & hsa04110 & Cell cycle & 23 & $8.06 e-05$ \\
KEGG pathway & hsa04978 & Mineral absorption & 12 & $2.43 e-04$ \\
KEGG pathway & hsa05410 & Hypertrophic cardiomyopathy (HCM) & 16 & $4.47 e-04$ \\
KEGG pathway & hsa04260 & Cardiac muscle contraction & 15 & $9.36 e-04$ \\
KEGG pathway & hsa04261 & Adrenergic signaling in cardiomyocytes & 22 & $1.00 e-03$ \\
KEGG pathway & hsa05414 & Dilated cardiomyopathy & 16 & $1.01 e-03$ \\
\hline
\end{tabular}

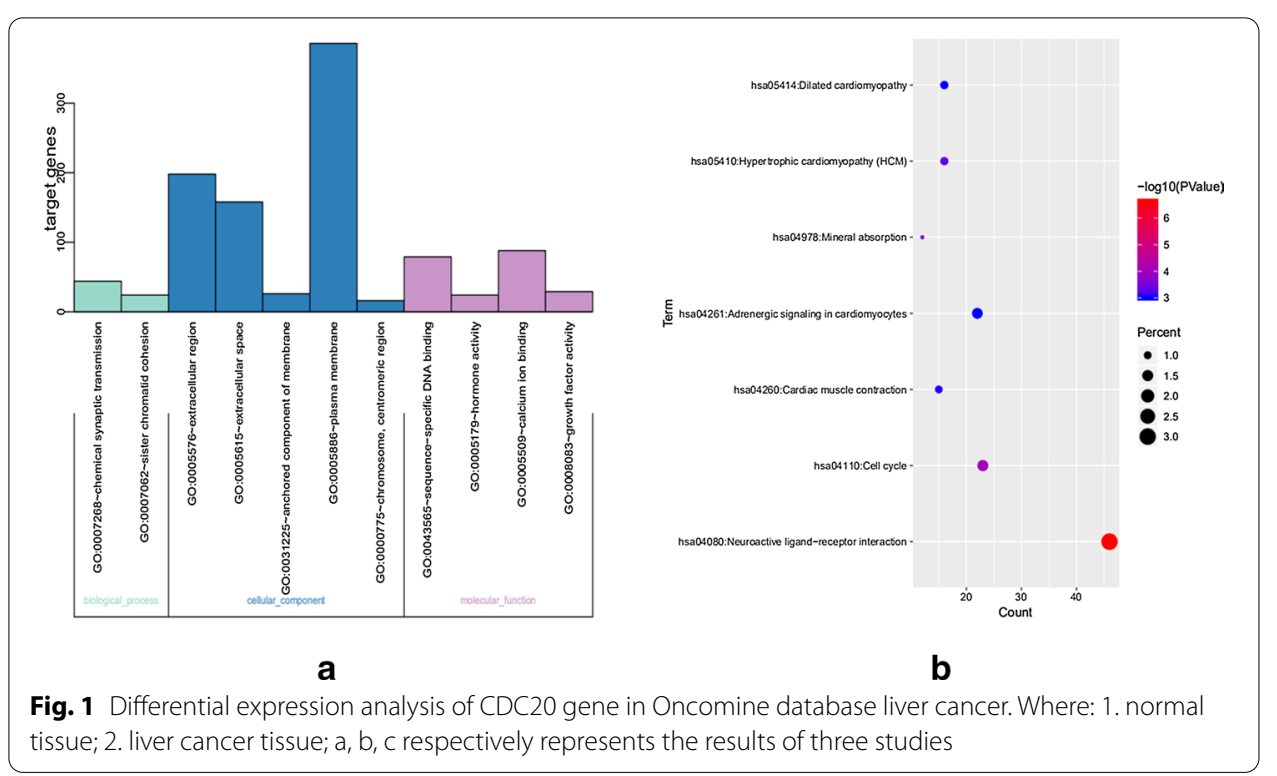

CCNA2, which have significant expression differences, as shown in Fig. 1. Furthermore, a meta-analysis of these 6 key genes was carried out. Taking CDC20 gene as an example, the results of differential expression analysis and meta-analysis are shown in Fig. 2. In the meta-analysis, three studies were screened by significant differences and differential expression folds $[7,8]$. Depending on the statistical analysis of Oncomine database, 6 key genes were highly expressed in liver cancer compared with normal tissues, and the differences were statistically significant. Next, we used the 5 -fold crossvalidation method and three classifiers: decision tree(Dtree), random forest(RF), and support vector machine(SVM) to measure the classification performance of 6 key 


\section{of CDC20 Across 3 Analyses}

Over-expression

\section{Median Rank p-Value Gene \\ 182.0 1.15E-64 CDC20}

$$
\begin{array}{|l|l|l|}
\hline 1 & 2 & 3 \\
\hline
\end{array}
$$

\section{Legend}

1. Hepatocellular Carcinoma vs. Normal

3. Hepatocellular Carcinoma vs. Normal Wurmbach Liver, Hepatology, 2007

Roessler Liver, Cancer Res, 2010

2. Hepatocellular Carcinoma vs. Normal Roessler Liver 2, Cancer Res, 2010

\section{$1 \quad 5 \quad 1025 \quad 251051$
$\square \square \square \square \square$$\square \square$ Not measured}

The rank for a gene is the median rank for that gene across each of the analyses. The $p$-Value for a gene is its $p$-Value for the median-ranked analysis.

Fig. 2 Meta-analysis of Oncomine database of CDC20 gene in liver cancer

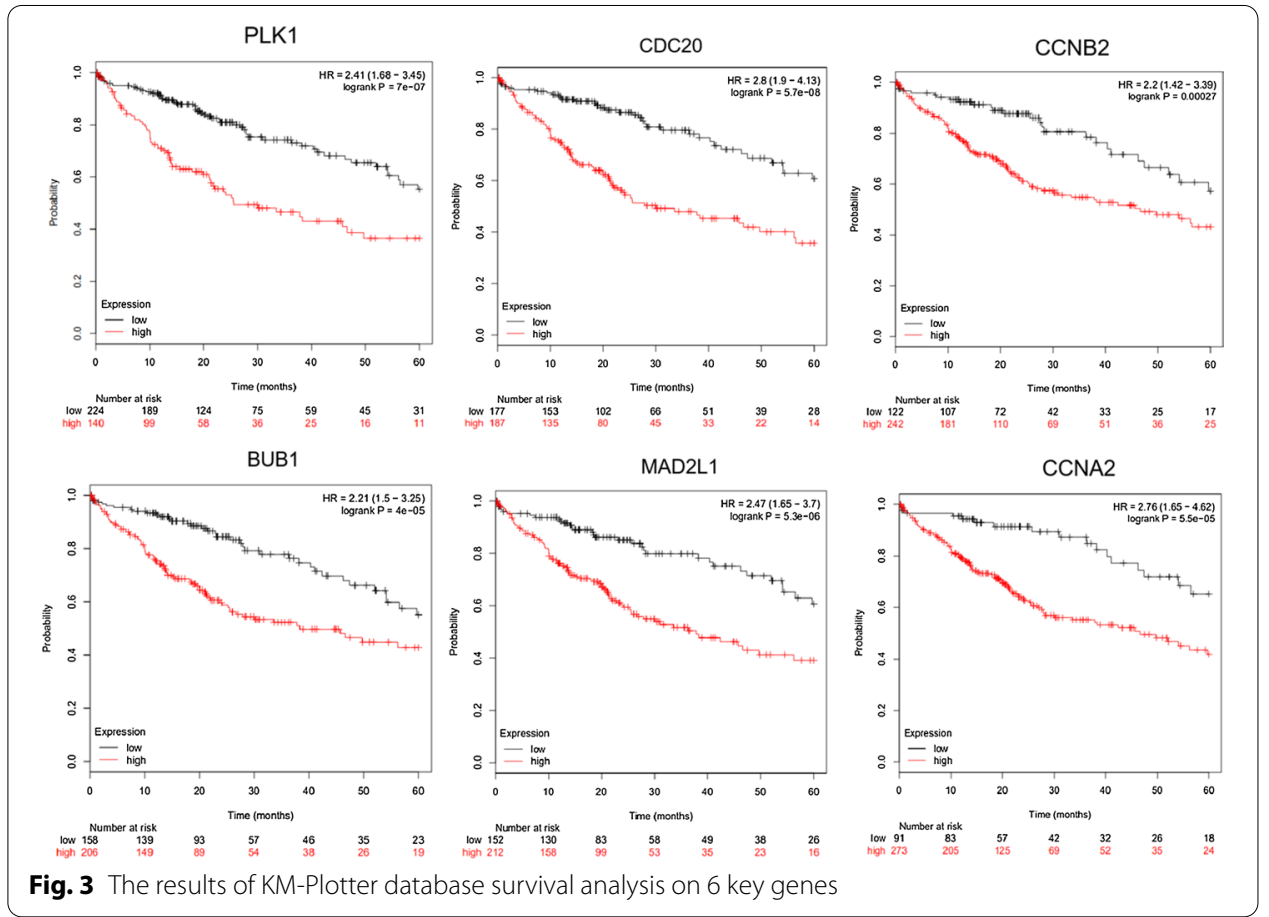

genes and 15 hub genes in 4 data sets. Taking into account the randomness of the classifier, the process was repeated 200 times and the average accuracy was calculated. The results are shown in Table 2.

Finally, using the KM-Plotter database, the survival curves were obtained based on the 6 key genes. Conducting research on the prognostic value of these key genes for the overall survival of patients with liver cancer, the results shown that: PLK1 $(P=7 e-07), \quad$ CDC20 $(P=5.7 e-08), \quad$ CCNB2 $(P=2.7 e-04), \quad$ BUB1 $(P=4 e-05)$, MAD2L1 $(P=5.3 e-06)$ and CCNA2 $(P=5.3 e-06)$. The analysis 


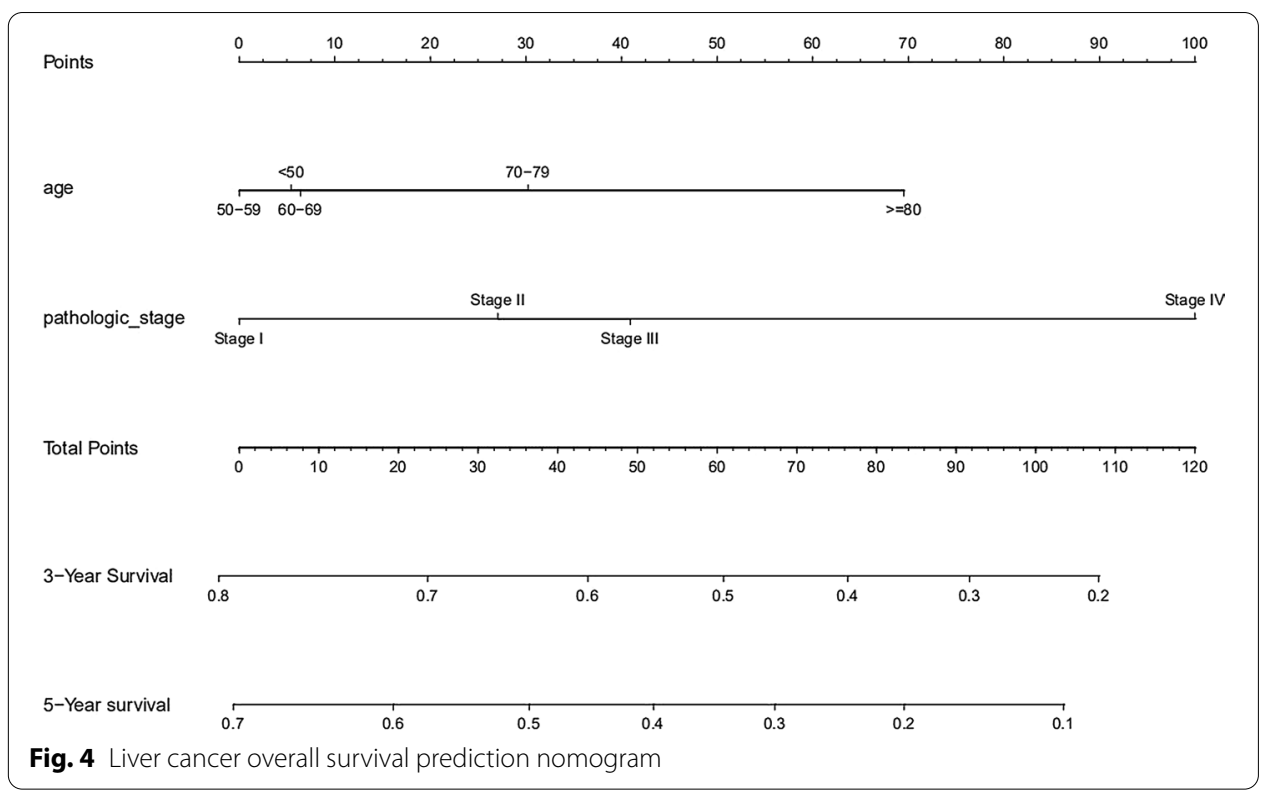

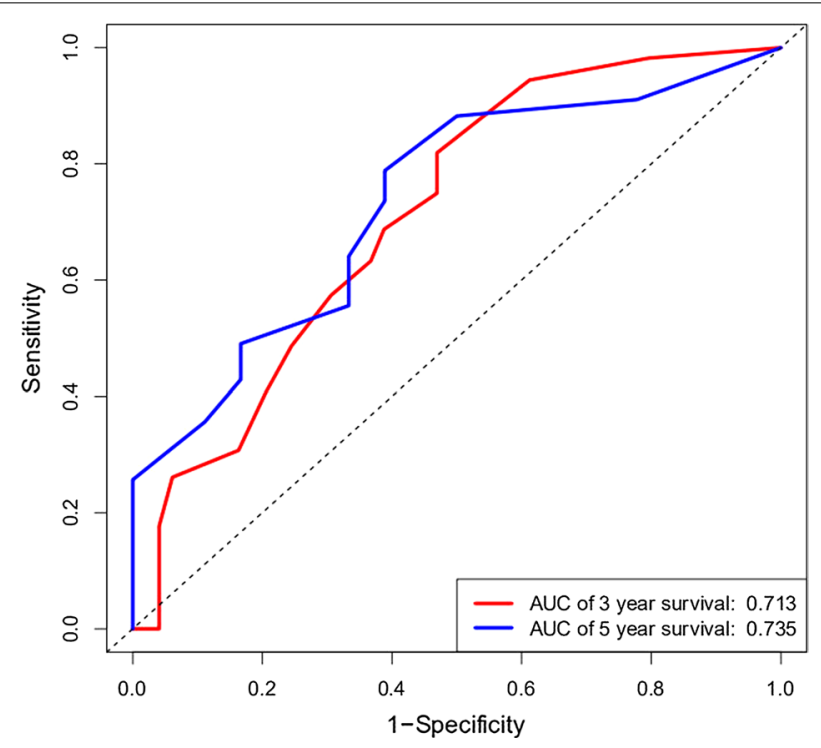

Fig. 5 ROC of the risk score

results of survival curve are shown in Fig. 3. These results show that these six key genes are closely related to the occurrence, development and prognosis of liver cancer, which were significant for the study of liver cancer.

Based on the clinical prediction model, a univariate cox regression analysis was performed on the training group data set, and the $\mathrm{p}$ value of age and pathology was less than 0.05. Multivariate cox regression analysis was performed for these two factors, and a nomogram was drawn as shown in Fig. 4. The ROC curve of the training 
Table 2 The classification accuracy of 15 hub genes and 6 key genes using 3 classifiers in 4 data sets

\begin{tabular}{lllll}
\hline Dataset & Number of genes & \multicolumn{3}{l}{ Classifier and classification accuracy } \\
\cline { 3 - 5 } & & Dtree & RF & SVM \\
\hline GSE76427 & 15 hub & 0.850 & 0.919 & 0.832 \\
& 6key & 0.878 & 0.907 & 0.827 \\
GSE57957 & 15 hub & 0.894 & 0.917 & 0.861 \\
& Gkey & 0.893 & 0.908 & 0.856 \\
GSE39791 & 15 hub & 0.916 & 0.911 & 0.887 \\
& Gkey & 0.925 & 0.915 & 0.915 \\
GSE102079 & 15 hub & 0.854 & 0.888 & 0.898 \\
& 6key & 0.843 & 0.859 & 0.875 \\
\hline
\end{tabular}

group (seen in Fig. 5) and the validation results of the test population show that the model has good prediction ability.

\section{Discussions}

It is a dynamic biological process with multiple molecules,steps and factors during the occurrence and development of liver cancer, and people's understanding of its mechanism is still very limited presently. Previous studies mainly focused on the impact of single genes on tumors, but the process of cell carcinogenesis often involves changes in multiple genes. These genes can play a role by transforming network interactions. Therefore, studying cancer gene expression profile at the level of multiple genes can help us better explore the pathogenesis. Gene chip can detect the expression levels of tens of thousands of genes at the same time, and is a powerful tool for studying the interaction between genomes and genes.

In this study, to verify the performance of the 6 key genes in classifying cancer and normal samples, four datasets from different sources were applied. By using different classifiers for verification, higher classification accuracy has been obtained. By comparing the classification accuracy of 15 hub gene sets and 6 key gene sets, we can find that the prediction accuracy of the two gene sets is not much different, but because the number of genes was reduced, the speed of clinical diagnosis can be improved. Therefore, our method has strong robustness and effectiveness. Compared with normal samples all of the 6 key genes are upregulated. Of the 6 key genes, PLK1 plays an important role in the regulation of cell mitosis and is closely related to survival and prognosis. In recent years, it has been found that PLK1 is highly expressed in cervical cancer [9], neuroblastoma cells [10], acute myeloid leukemia [11], prostate cancer [12] and many other malignant tumors. Studies have found that inhibiting the expression of PLK1 by interfering with multiple stages of mitosis can lead to tumor cell death, so PLK1 is expected to become a potential target for cancer treatment [13].

$\mathrm{CDC} 20$ is a key gene to ensure the normal progress of cell mitosis. Its abnormal expression can cause normal cells to produce non-integer chromosomes by disrupting 
cell mitosis, thereby promoting malignant transformation of cells [14]. Studies have shown that the high expression of CDC20 is closely related to the clinical progress of human malignant tumors $[15,16]$, such as lung cancer, liver cancer, malignant glioma, etc [17-19].

The proteins encoded by CCNA2 and CCNB2 belong to the highly conserved cyclin family and play an important role in the process of cell cycle control. The research results of the literature $[20,21]$ show that they are all highly expressed in HCC tissues, and the overall survival rate of patients is low.

BUB1 encodes a serine/threonine-protein kinase that play an important role in mitosis [22]. The protein partly encoded by this gene regulates the formation and activation of the late mitotic complex by phosphorylating some components of the mitotic checkpoint complex and activating the checkpoint of the spindle [23, 24]. The abnormal expression of BUB1 is related to the occurrence and development of a variety of tumors, and it is also involved in the biological behavior of cancer stem cells such as breast cancer $[25,26]$.

MAD2L1 is an important part of the spindle assembly checkpoint in mitosis. Studies have shown that reducing the expression of MAD2L1 can inhibit tumor cell migration and invasion, thereby hindering tumor occurrence and development [27].

In the process of cell carcinogenesis, the interaction of multiple gene changes is of great significance to the pathogenesis of cancer, not only for liver cancer. Therefore, the analysis method in this study is also applicable to other cancers and is used to identify biomarkers of other complex human diseases, such as the non-coding RNA, microRNAs, etc [28-30].

\section{Conclusions}

This study comprehensively explored the molecular functions and biological processes of the occurrence and development of liver cancer. Based on the analysis of the liver cancer data from the TCGA database, firstly compared the gene expression of normal tissues and liver cancer tissues, and screened out genes with different expressions. The GO enrichment analysis and KEGG pathway enrichment analysis of these differential genes shown that DEGs were mainly enriched in chemical synaptic transmission, extracellular area, extracellular space, neuroactive ligand-receptor Interaction, cell cycle, etc. Then, the PPI network of DEGs was constructed using STRING database and Cytoscape software, and 15 hub genes were screened out. In addition, in order to verify the expression levels of these key genes, differential expression analysis and meta-analysis of 15 hub genes were performed using Oncomine database, and 6 key genes were obtained, and the fivefold verification method was used to verify the effectiveness of these six key genes. Finally, Oncomine and survival analysis were used to confirm the six key genes that PLK1, CDC20, CCNB2, BUU1, MAD2L1 and CCNA2 are closely related to the occurrence, prognosis and mechanism of liver cancer, which will help us discover tumor markers and drug targets. 


\section{Methods}

\section{Datasets}

The mRNA data of liver cancer tissues and normal tissues were downloaded from the TCGA database. The data includes 371 tumor samples and 50 normal samples. The four gene expression profiles (GSE76427 [31], GSE57957 [32], GSE39791 [33], GSE102079 [34]) used for the verification test were obtained from the Gene Expression Comprehensive System (GEO) [35] Download.

\section{Methods}

This paper aims to find molecular markers for early diagnosis of liver cancer and potential molecular targets for liver cancer immunotherapy. The methods used in the study were as follows: Screening for the DEGs, GO and KEGG enrichment analysis, PPI network construction and hub genes identification, Oncomine validation and survival analysis of hub genes. These methods were described in detail as follows:

\section{Screening for the DEGs}

Using the $\mathrm{R}$ package, we performed background correction, standardization and expression value calculation on the original data. Index $|\log F C| \alpha$ and $F D R<\beta$ were used as conditions to filter out DEGs, where the value of $\alpha$ was inversely proportional to the number of DEGs selected, and the value of $\beta$ was proportional to the number of DEGs selected.

\section{GO and KEGG enrichment analysis}

GO is a method for large-scale enrichment studies of gene functions including biological processes, molecular functions, and cellular components. Statistical analysis was used to calculate the $p$ value and FDR in GO. KEGG pathway enrichment analysis calculates the hypergeometric distribution of DEGs and pathways, and returns the $p$ value and FDR value of each pathway existing in DEGs to find the most likely related pathway. In this study, we used DAVID 6.8 database for GO and KEGG enrichment analysis. Then visually analyze GO and KEGG through bar graphs and ggplot2.

\section{PPI network construction and hub genes identification}

The STRING database is an online analysis tool used to study protein-protein interaction (PPI) patterns and provide information on related pathways and functions [36]. Import DEGs into the database and output network files of protein interactions. Then use the Cytoscape software to visualize the protein interaction network and obtain the hub genes $[37,38]$.

\section{Oncomine validation and survival analysis of hub genes}

The Oncomine database is a cancer gene chip database used to analyze cancer gene information, compare the differential expression between cancer and normal tissues, and verify hub genes. The KM-plotter database (http://kmplot.com/analysis/) contains information on 364 liver cancer patients and is used to evaluate the prognostic value of hub genes in liver cancer patients. 


\section{In silico validation}

In order to verify the validity of the results, we downloaded 4 independent gene expression profiles of GEO (GSE76427, GSE57957, GSE39791, GSE102079). The five-fold cross-classification verification method was adopted, and the four data sets were respectively verified using decision trees, random forests and support vector machines. Each experiment was repeated 200 times and the average classification accuracy was calculated.

\section{Construction of clinical prediction model}

The clinical data of liver cancer samples were randomly divided into training group and testing group. Univariate cox regression analysis was performed using the training group to screen out statistically significant factors and draw the histograms. According to the calculation results, the training group was divided into high-risk and low-risk categories, and the ROC curve was constructed to evaluate the model. The testing group was used to verify the model.

In this study, the DEGs were obtained by differential expression analysis of liver cancer gene expression profiles. These differential expression genes were studied by GO and KEGG enrichment analysis, and the significantly enriched functions and pathways of DEGs were obtained. Furthermore, protein interaction network was constructed using STRING database to screen key genes. Oncomine database and survival analysis were used to confirm the key genes, and the genes which suitable for liver cancer markers or potential targets were obtained. Finally, we constructed a clinical prediction model, so as to get more convincing results.

Abbreviations

DEG: Differentially expressed genes; GO: Gene ontology; KEGG: Kyoto encyclopedia of genes and genomes; DAVID: The database for annotation, visualization and integrated discovery; PPI: Protein-protein interaction; FC: Fold change; FDR: False discovery rate; KM-Plotter: Kaplan-Meier Plotter database; STRING: STRING database.

\section{Acknowledgements}

This work was supported by the National Natural Science Foundation of China (Grand No. 11371174).

About this supplement

This article has been published as part of BMC Bioinformatics Volume 22 Supplement 3, 2021: Proceedings of the 2019 International Conference on Intelligent Computing (ICIC 2019): bioinformatics. The full contents of the supplement are available online at https://bmcbioinformatics.biomedcentral.com/articles/supplements/volume-22-supplement-3.

\section{Authors' contributions}

XQT designed the study. YCW and ZBT has major contribution in data gathering, algorithm design and experiment. YCW and ZBT wrote the daft. All authors have read and approved the final manuscript.

\section{Funding}

This is an exploratory research work on data processing methods. Except for the publication cost of this study funded by the National Natural Science Foundation of China (Grand No. 11371174), no funding body played any role in the design of the study and collection, analysis, and interpretation of data and in writing the manuscript.

Availability of data and materials

Not applicable.

\section{Declarations}

Ethics approval and consent to participate

Not applicable.

Consent for publication

Not applicable. 


\section{Competing interests}

The authors declare that they have no competing interests.

\section{Author details}

${ }^{1}$ School of Science, Jiangnan University, Wuxi 214122, China. ${ }^{2}$ Wuxi Engineering Research Center for Biocomputing, Wuxi 214122, China.

Received: 22 September 2021 Accepted: 24 September 2021

Published online: 25 October 2021

\section{References}

1. Chen W. Cancer statistics: updated cancer burden in China. Chin J Cancer Res. 2015;27(1):1.

2. Erstad DJ, Tanabe KK. Hepatocellular carcinoma: early-stage management challenges. J Hepatocell Carcinoma. 2017:4:81-92.

3. Gong YZ, Jiang YA. Significance of abnormal gene expression in the pathogenesis and development of hepatocelIular carcinoma in its diagnosis and treatment. J Gastroenterol Hepatol. 2016;25(8):848-51.

4. Zhou YL, Liu J, Li YL, et al. Analysis of prognosis and mechanism of CENPN in liver hepatocellular carcinoma based on bioinformatics. Chin J Immunol. 2021.

5. Wang P, Wang YC, Yu DD, et al. Expression of microRNA-1246 in the serum of hepatocellular carcinoma and its effect on biological function of HepG2 cells. Chin Gen Practice. 2021;19(08):1292-5.

6. Rhodes DR, Kalyana-Sundaram S, Mahavisno V. Oncomine 3.0: genes, pathways, and networks in a collection of 18,000 cancer gene expression profiles. Neoplasia. 2007;9(2):166-80.

7. Roessler S, Jia HL, Budhu A. A unique metastasis gene signature enables prediction of tumor relapse in early-stage hepatocellular. Carcinoma Patients Cancer Res. 2010;70(24):10202-12.

8. Wurmbach E, Ying-bei C, Khitrov G. Genome-wide molecular profiles of HCV-induced dysplasia and hepatocellular carcinoma. Hepatology. 2007;45(4):938-47.

9. Yang X, Chen G, Li W. Cervical cancer growth is regulated by a C-ABL-PLK1 signaling axis. Can Res. 2016;77(5):1142-54

10. Pajtler KW, Sadowski N, Ackermann S. The GSK461364 PLK1 inhibitor exhibits strong antitumoral activity in preclinical neuroblastoma models. Oncotarget. 2016;8(4):6730-41.

11. Tao YF, Li ZH, Du WW. Inhibiting PLK1 induces autophagy of acute myeloid leukemia cells via mammalian target of rapamycin pathway dephosphorylation. Oncol Rep. 2017;37(3):1419-29.

12. Li J, Wang R, Kong Y. Targeting Plk1 to enhance efficacy of Olaparib in castration-resistant prostate cancer. Mol Cancer Ther. 2017;16(3):469-79.

13. Liu Z, Sun Q, Wang X. PLK1, a potential target for cancer therapy. Transl Oncol. 2016;10(1):22.

14. Huang HC, Shi J, Orth JD. Evidence that mitotic exit is a better cancer therapeutic target than spindle assembly. Cancer Cell. 2009;16(4):347-58.

15. Wu WJ, Hu KS, Wang DS. CDC20 overexpression predicts a poor prognosis for patients with colorectal cancer. J Transl Med. 2013;11(1):142.

16. Wang Z, Wan L, Zhong J. A potential novel therapeutic target for cancer treatment. Curr Pharm Des. 2013;19(18):3210-4.

17. Shi R, Sun Q, Sun J. Cell division cycle 20 overexpression predicts poor prognosis for patients with lung adenocarcinoma. Tumor Biol. 2017;39(3):101042831769223.

18. Kim HS, Vassilopoulos A, Wang RH. SIRT2 maintains genome integrity and suppresses tumorigenesis through regulating APC/C activity. Cancer Cell. 2011;20(4):487-99.

19. Xie Q, Wu Q, Mack SC. CDC20 maintains tumor initiating cells. Oncotarget. 2015;6(15):13241-54

20. Gao C, Wang G, Yang G. Karyopherin subunit- $\alpha 2$ expression accelerates cell cycle progression by upregulating CCNB2 and CDK1 in hepatocellular carcinoma. Oncol Lett. 2017;15(3):2815-20.

21. Gopinathan L, Tan SLW, Padmakumar VC. Loss of Cdk2 and Cyclin A2 impairs cell proliferation and tumorigenesis. Can Res. 2014;74(14):3870-9.

22. Akhoundi F, Parvaneh N, Modjtaba EB. In silico analysis of deleterious single nucleotide polymorphisms in human BUB1 mitotic checkpoint serine/threonine kinase B gene. Meta Gene. 2016;9:142-50.

23. Ricke RM, Jeganathan KB, Van Deursen JM. Bub1 overexpression induces aneuploidy and tumor formation through Aurora B kinase hyperactivation. J Cell Biol. 2011;193(6):1049-64.

24. Jia L, Li B, Yu H. The Bub1-CPIk1 kinase complex promotes spindle checkpoint signalling through Cdc20 phosphorylation. Nat Commun. 2016;7:10818.

25. Chang JT, Reiner SL. lessons from lymphocytes. Cold Spring Harb Symp Quant Biol. 2008;73:73-9.

26. Lathia JD, Hitomi M, Gallagher J. Distribution of CD133 reveals glioma stem cells self-renew through symmetric and asymmetric cell divisions. Cell Death Dis. 2011;2:e200.

27. Nascimento AV, Singh A, Bousbaa H. Overcoming cisplatin resistance in non-small cell lung cancer with Mad2 silencing siRNA delivered systemically using EGFR-targeted chitosan nanoparticles. Acta Biometarialia. 2017:47:71-80.

28. Chen X, Wang L, Qu J, Guan NN, Li JQ. Predicting miRNA-disease association based on inductive matrix completion. Bioinformatics. 2018;34(24):4256-65.

29. Chen X, Xie D, Zhao Q, You ZH. MicroRNAs and complex diseases: from experimental results to computational models. Brief Bioinform. 2019;20(2):515-39.

30. Chen X, Yin J, Qu J, Huang L. MDHGl: matrix decomposition and heterogeneous graph inference for miRNA-disease association prediction. PLoS Comput Biol. 2018;14(8):e1006418. 
31. Grinchuk OV, Yenamandra SP, Iyer R, et al. Tumor-adjacent tissue co-expression profile analysis reveals pro-oncogenic ribosomal gene signature for prognosis of resectable hepatocellular carcinoma. Mol Oncol. 2018;12(1):89-113.

32. Mah WC, Thurnherr T, Chow PK, et al. Methylation profiles reveal distinct subgroup of hepatocellular carcinoma patients with poor prognosis. PLoS One. 2014;9(8):e104158.

33. Kim JH, Sohn BH, Lee HS, et al. Genomic predictors for recurrence patterns of hepatocellular carcinoma: model derivation and validation. PLoS Med. 2014;11(12):e1001770.

34. Chiyonobu N, Shimada S, Akiyama Y, et al. Fatty acid binding protein 4 (FABP4) overexpression in intratumoral hepatic stellate cells within hepatocellular carcinoma with metabolic risk factors. Am J Pathol. 2018;188(5):1213-24.

35. Barrett T, Wilhite SE, Ledoux P, et al. NCBI GEO: archive for functional genomics data sets-update. Nucleic Acids Res. 2013;41:D991-5.

36. Szklarczyk D, Franceschini A, Wyder S. STRING v10: protein-protein interaction networks, integrated over the tree of life. Nucleic Acids Res. 2015;43(D1):D447-52.

37. Shannon P. A software environment for integrated models of biomolecular interaction networks. Genome Res. 2003;13(11):2498-504.

38. Chin $\mathrm{CH}$, Chen $\mathrm{SH}$, Wu HH. Identifying hub objects and sub-networks from complex interactome. BMC Syst Biol. 2014:8(Suppl 4):S11-S11.

\section{Publisher's Note}

Springer Nature remains neutral with regard to jurisdictional claims in published maps and institutional affiliations.

- fast, convenient online submission

- thorough peer review by experienced researchers in your field

- rapid publication on acceptance

- support for research data, including large and complex data types

- gold Open Access which fosters wider collaboration and increased citations

- maximum visibility for your research: over $100 \mathrm{M}$ website views per year

At BMC, research is always in progress.

Learn more biomedcentral.com/submissions 